\title{
Numerical simulation of leakage flow field and acoustic characteristics for safety valve
}

\author{
Fan Qian ${ }^{1}$, and Minghui $\mathrm{Hu}^{1, *}$ \\ ${ }^{1}$ Key Laboratory of Pressure Systems and Safety, East China University of Science and Technology, \\ 200237, Shanghai, China
}

\begin{abstract}
Aiming at the internal leakage problem of spring type nuclear safety valve at the sealing surface, the flow field and sound field characteristics at the leakage height of $0.5 \mathrm{~mm}$ between the valve disc and the valve seat sealing surface were studied, and the numerical simulation was carried out based on large eddy simulation(LES) and mohring acoustic analogy method, and compare the effects of acoustic wall pressure fluctuation(AWPF) and turbulent wall pressure fluctuation(TWPF) as the excitation source on the external sound field of the valve. The simulation results show that: the change gradient of velocity field and pressure field at the leakage port of safety valve is significant and form vortices of different sizes. The small-scale vortices are mainly in the leakage port, while the large-scale vortices mainly exist in the flow channel; When the valve is leaking, the noise is mainly dominated by high-pressure injection noise, its spectrum curve shows wide-band characteristics, and the external noise of the valve is mainly caused by AWPF. The above research results can provide a theoretical basis for the safety valve online detection method.
\end{abstract}

\section{Introduction}

Nowadays, the safety valve is widely used in various fields. As an overpressure protection device, internal leakage is the main failure mode. When the internal leakage of safety valve occurs, it will not only cause serious accidents, but also cause great losses to enterprises. Therefore, how to accurately and quickly detect valve internal leakage has become a hot topic of current research ${ }^{[1]}$.

At present, there are more and more technologies for valve leakage detection at home and abroad, such as acoustic emission testing, vibration analysis and ultrasonic testing methods ,etc ${ }^{[2,3]}$. The paper ${ }^{[4,5]}$ used CFD and acoustic software to study the flow field and aerodynamic noise problems during valve internal leakage, and verified the feasibility of this method. Juhani et al ${ }^{[6-8]}$ carried out experimental research on valve internal leakage by using acoustic emission detection technology, which proved that the valve internal leakage can be well detected by acoustic emission detection technology. Ma Haodong et $\mathrm{al}^{[9,10]}$ used numerical simulation and experimental valve internal leakage to study, and established a quantitative relationship between various parameters and leakage.

In this paper, a spring safety valve with a leakage height of $0.5 \mathrm{~mm}$ between the valve disc

Corresponding author: agile_hu@ecust.edu.cn 
and the seat sealing surface is taken as the research object. Based on the LES and mohring acoustic analogy, the variation of velocity field and pressure field as well as the frequency spectrum characteristics of internal and external noise of the valve are analyzed, which provides a certain data basis for establishing the relationship between noise and leakage, and also for the leakage acoustics inside the safety valve Online monitoring provides a theoretical basis.

\section{Basic theory}

LES theory divides turbulence into two kinds of motion: large-scale vortex and small-scale vortex, in which large-scale vortex dominates the transport of overall momentum and energy, while small-scale vortex is mainly responsible for dissipation of turbulent kinetic energy. The filtered large eddy simulation equation is:

$$
\begin{gathered}
\frac{\partial \bar{u}_{i}}{\partial x_{i}}=0 \\
\frac{\partial}{\partial_{t}}\left(\rho \overline{u_{i}}\right)+\frac{\partial}{\partial x_{j}}\left(\rho \bar{u}_{i} \bar{u}_{j}\right)=\frac{\partial}{\partial x_{j}}\left(u \frac{\partial \sigma_{i j}}{\partial x_{j}}\right)-\frac{\partial \bar{p}}{\partial x_{i}}-\frac{\partial \tau_{i j}}{\partial x_{j}}
\end{gathered}
$$

where, $\bar{u}_{i}$ refer to filtered average velocity component, $\sigma_{i j}$ refer to stress tensor caused by molecular viscosity, and $\tau_{i j}$ represents the subgrid Reynolds stress.

$$
\tau_{i j}=\rho \overline{u_{i} u_{j}}-\rho \overline{u_{i} u_{j}}
$$

The Mohring model considers the influence of the convection effect sound field, and its sound source term is composed of bulk sound source $R_{V}$ and surface sound source $R_{S}{ }^{[11]}$, the expression is as follows:

$$
\begin{gathered}
R_{V}=\int_{V} \frac{\partial N_{a}}{\partial x} F F T\left[\frac{\rho}{\rho_{T}}\left(v w-\frac{\partial \tau_{i j}}{\partial x_{j}}-\frac{u}{\rho_{T}} \frac{\partial \rho}{\partial s}\right)_{p} \frac{\partial s}{\partial t}+\frac{\rho T}{\rho_{T}} \frac{\partial s}{\partial x}\right] d V+i \omega F F T\left\{\int_{V}\left[\frac{N_{a}}{\rho_{T}}\left(\frac{\partial \rho}{\partial s}\right)_{p} \frac{\partial s}{\partial t}-\frac{\rho u N_{a}}{\rho_{T}^{2}} \frac{\partial \rho_{T}}{\partial x}\right] d V\right\} \\
R_{s}=i \omega F F T\left(\int_{V} N_{a} \frac{\rho u \bullet n_{i}}{\rho_{T}} d s\right)
\end{gathered}
$$

where, $N_{a}$ is the shape function; $\rho$ is the static density, $\rho_{T}$ is the stagnation density; $\tau_{i j}$ is the viscous stress tensor; $\partial \tau_{i j} / \partial x_{j}, \partial s / \partial t, \partial \rho_{T} / \partial x$ refer to viscous effect, entropy change and density fluctuation, which can be ignored for the high-speed flow field.

\section{3 model and calculation scheme of safety valve}

\section{1 physical model}

The three-dimensional structure of the safety valve is shown in Fig.1. The inlet diameter of the safety valve is $82.36 \mathrm{~mm}$, the throat diameter is $57.8 \mathrm{~mm}$, the valve disc seat diameter is $125 \mathrm{~mm}$, and the outlet diameter is $200 \mathrm{~mm}$. 


\subsection{Flow field calculation}

Because the safety valve is symmetrical structure, only half of the model is selected as the computational fluid domain to reduce the calculation amount. The safety valve is divided into five parts: inlet, disc, body, hole and outlet. Structured hexahedral mesh generation by ICEM, and the total number of mesh is about 1 million, as shown in Fig.2.

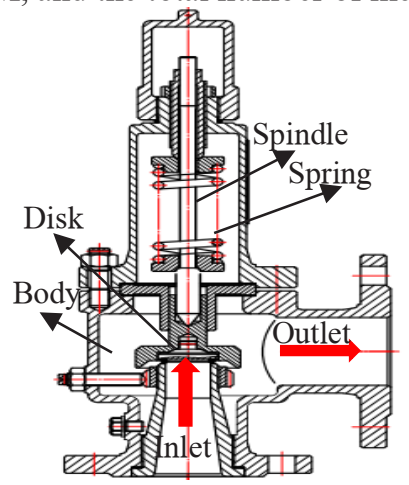

Fig.1. Structure of spring safety valve.

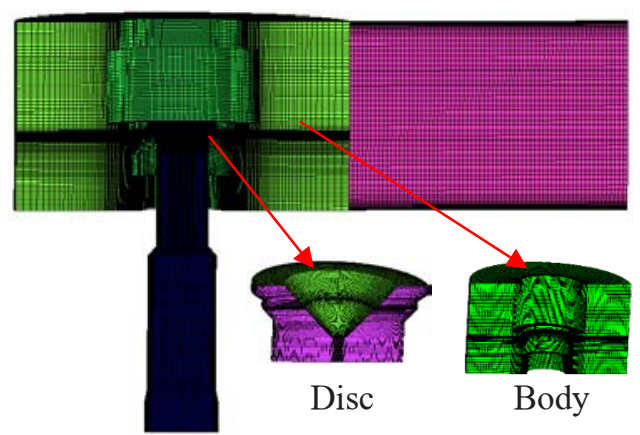

Fig.2. Meshing of spring safety valve.

In this paper, saturated vapor is used as the fluid medium, the RNG k- $\varepsilon$ turbulence model is adopted, and the transient time step is set to $5 \times 10^{-6} \mathrm{~s}$, pressure inlet and pressure outlet are chosen as the boundary condition. The inlet pressure is $2 \mathrm{Mpa}$, the temperature is $485.57 \mathrm{~K}$, and the outlet pressure is atmospheric pressure and wall is set as no-slip boundary condition.

\subsection{Sound field calculation}

Since the noise is mainly concentrated near the hole and the outlet, the inlet model is omitted. As shown in Fig.3, the sound field model is mainly composed of sound source zone, sound propagation zone and infinite element surface. The sound source area uses the integral difference method ${ }^{[12]}$ to interpolate the results of the transient flow field to the acoustic mesh, and the infinite element surface is used to set the non-reflective boundary conditions. The unstructured tetrahedral mesh is used, and the maximum mesh size is $10 \mathrm{~mm}$. Fig.4shows the setting of the positions of different monitoring points during the sound field calculation.
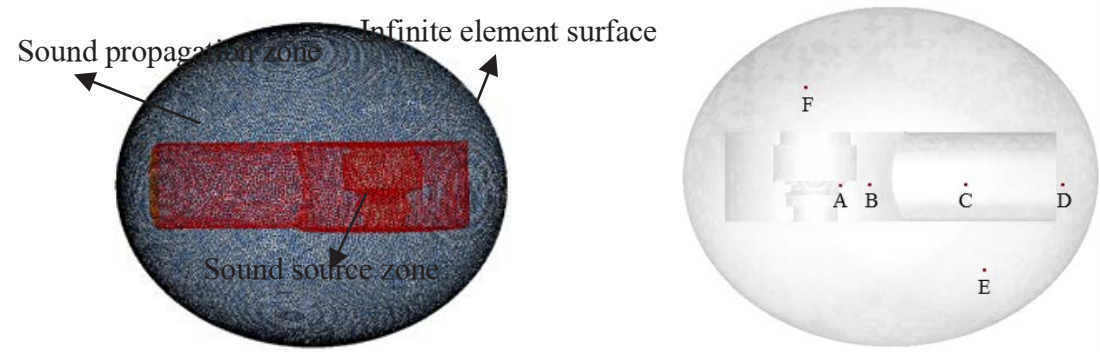

Fig.3. Acoustic computational grid model.

Fig.4. Acoustic calculation monitoring point setting.

\section{Results and analysis}

\subsection{Simulation results and analysis of flow field}

The pressure distribution nephogram of transient flow field on the symmetry plane, as shown 
in Fig.5. It can be seen that the pressure changes little at the inlet, valve disc and outlet, but there is an obvious pressure change gradient at the leakage outlet, the pressure rapidly drops from $2 \mathrm{MPa}$ to $0.3 \mathrm{MPa}$, which is due to the flow area of saturated vapor is greatly reduced when passing through the leakage hole, produces a process of adiabatic expansion, resulting in a decrease in pressure. The velocity vector at a certain moment is shown in Fig. 6.Due to the throttling effect, the velocity of saturated steam increases rapidly at the leakage port, resulting in high-speed jet, with the maximum speed reaching $1154 \mathrm{~m} / \mathrm{s}$, some of the jet flow to both sides of the valve body, forming vortex at the boundary, and the other part is fully developed in the outlet pipe to formed stable flow.

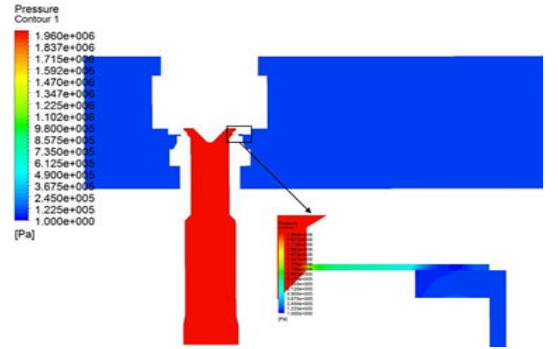

Fig.5. Pressure distribution nephogram of flow filed

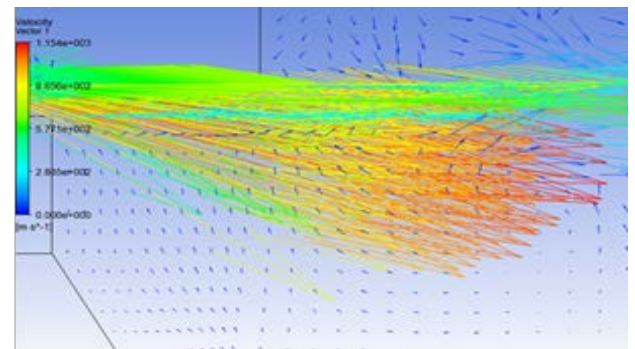

Fig.6. Velocity vector diagram

The distribution of vorticity at different times, as shown in Fig.7. It can be seen that the maximum value of vorticity is $0.6-0.8 \mathrm{~m}$ away from the leakage port, which is caused by the shear action of saturated steam ejected from high-speed jet. During the whole period, it can be seen that there are mainly small-scale vortices near the leakage hole. With the continuous generation, shedding and development of vortices, they gradually develop into large-scale vortices in the downstream. And these vortices also have a great relationship with the safety valve noise ${ }^{[13]}$.

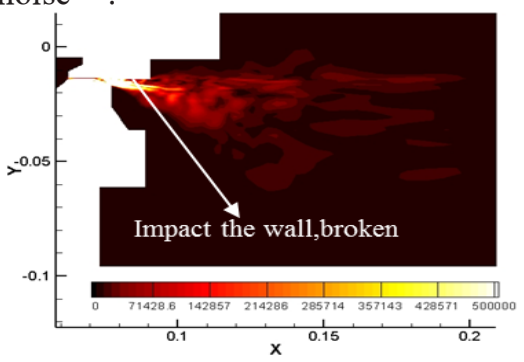

(a) $\mathrm{T} 1=0.015$

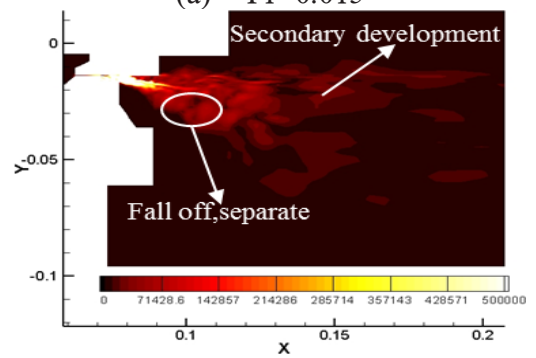

(c) $\mathrm{T} 3=0.0152$

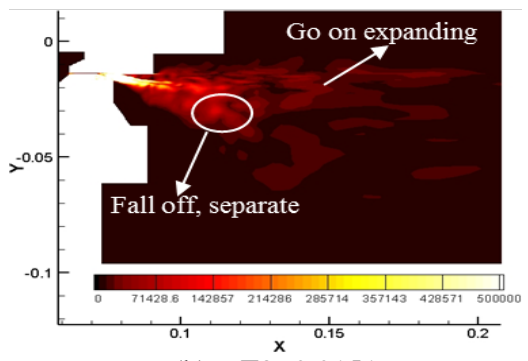

(b) $\mathrm{T} 2=0.0151$

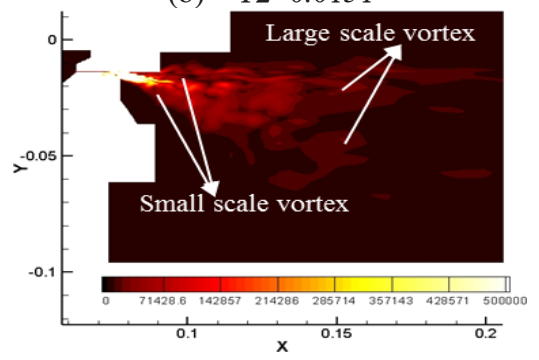

(d) $\mathrm{T} 4=0.0153$

Fig.7. Instantaneous vorticity diagram at different times.

\subsection{Simulation results and analysis of sound field}

Fig. 8 shows the spectrum curve of sound pressure at different monitoring points, it can be 
seen that the changes of frequency spectrum curves at each monitoring point are basically the same, and the sound pressure level decreases with the increase of frequency. The sound pressure level of the monitoring point close to the leakage is the largest, and as the distance between the monitoring point and the leakage port is farther, the sound pressure level also decreases, which also indicates that the eddy current movement near the leakage port is more intense. Comparing the monitoring points $\mathrm{D}, \mathrm{E}, \mathrm{F}$, when the frequency is higher than $1500 \mathrm{HZ}$, the sound pressure change amplitude is lower and no more than $15 \mathrm{~dB}$, which is because the valve noise is mainly caused by the small-scale vortex at the leakage port at high frequency. Fig. 9 shows the 1/3octave diagram of different monitoring points, showing the characteristics of wide-band noise. The injection noise caused by valve leakage is mainly distributed in the low-frequency area below $1500 \mathrm{~Hz}$, and the sound pressure level drops rapidly in the middle and high-frequency area.

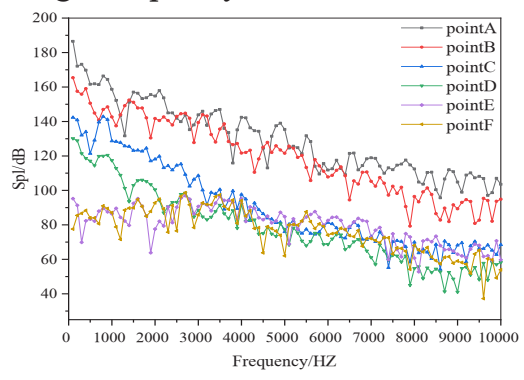

Fig. 8. Sound pressure spectrum curve.

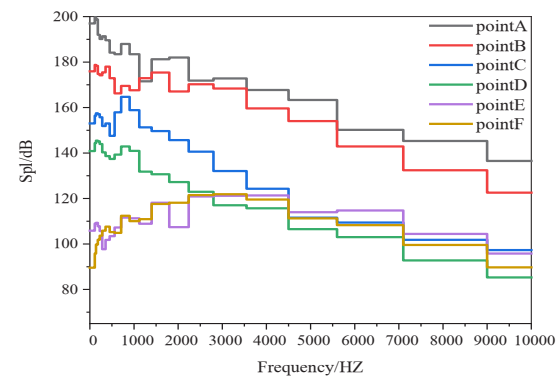

Fig. 9. One-third octave diagram.

The AWPF and TWPF are respectively loaded as excitation sources on the model shown in Fig. 3 to calculate the sound field outside the valve. The results are shown in Fig.10, it can be seen from the sound pressure spectrum curve of monitoring points $E$ and $F$. The external noise of the valve is the result of the joint action of the two pressure fluctuation, and the external noise generated by AWPF sound source is greater than that generated by TWPF at the same frequency.

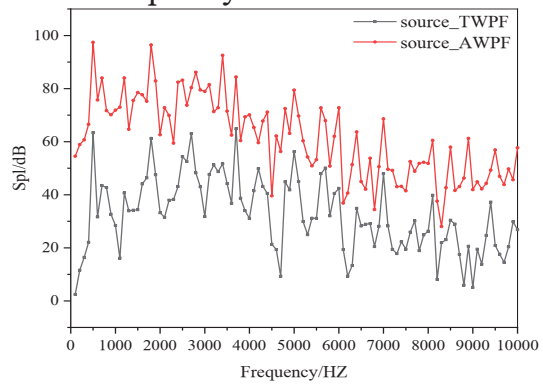

(a) Monitoring pointE

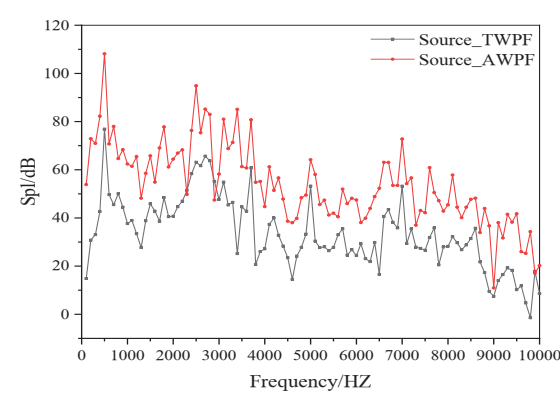

(b) Monitoring pointF

Fig. 10. Spectrum curves of different sound sources at external monitoring points.

Fig.11 shows the internal sound pressure level cloud diagram of the safety valve, which further verifies the results of the above vorticity variation. The low-frequency noise is mainly generated by the small-scale vortex near the leakage port, and the high-frequency noise is mainly generated by the large-scale vortex at the downstream. It can be seen from the Fig. 12 that when the frequency is lower than $1500 \mathrm{~Hz}$, the external noise mainly diffuses to the outside in an arc at the outlet. When the frequency is higher than $1500 \mathrm{~Hz}$, the external noise of the valve diffuses to the upper part of the valve in an oval shape near the leakage port. 


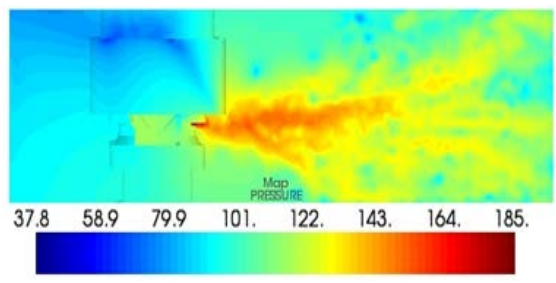

(a) $1500 \mathrm{HZ}$

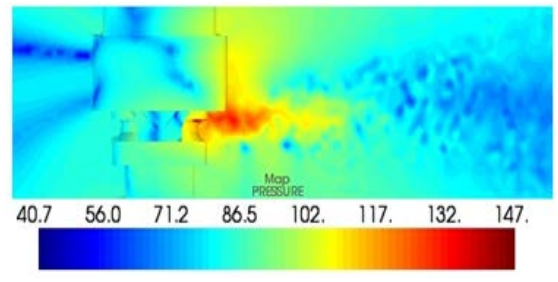

(b) $5500 \mathrm{HZ}$

Fig. 11. Cloud chart of internal sound pressure level of safety valve.

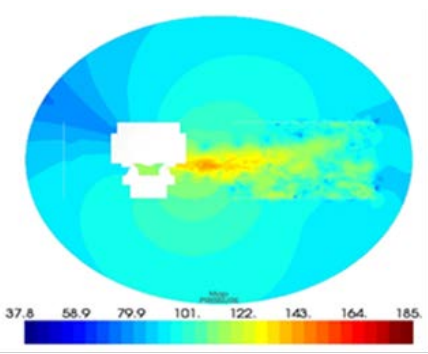

(a) $1500 \mathrm{HZ}$

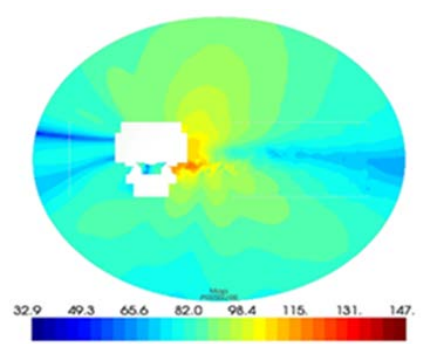

(b) $5500 \mathrm{HZ}$

Fig. 12. Overall sound pressure level nephogram of safety valve.

\section{Conclusion}

In this paper, numerical simulation method is used to study the flow field and acoustic characteristics of safety valve when internal leakage occurs. The flow field of safety valve is calculated by Fluent. The results show that when saturated vapor passes through the leak, both the pressure field and the velocity field will change significantly due to the change of flow area. Small-scale vortices will be generated near the leak and large-scale vortices will be generated downstream of the leak. Based on mohring acoustic analogy method, the sound field of safety valve is calculated by using the sound source information obtained in flow field calculation. It is concluded that the noise of valve leakage is mainly the high-pressure jet noise generated by saturated steam at the leakage port, the noise shows wide-band characteristics, and the injection noise is mainly distributed in the low frequency area below $1500 \mathrm{HZ}$. The external noise of the valve is mainly produced by the interaction of two kinds of wall pressure fluctuation, but AWPF noise source is dominant in the valve external noise.

This study was supported National Natural Science Foundation of China (Grant No.51775188) and National Key R\&D Program of China (Grant No.2018YFC1902404).

\section{References}

1. G.Dai, Y.T.Xu, W.Li, et al, Jouranl of northeast Prtroleum University, 2001, 25(3):95-98.

2. M. M. Wu, X.C. Dong, Y.D. Zhao, et al, Journal of Shandong University of Science and Technology(Natural Science), 2019, 38(06):105-113.

3. J.C. Zhou, Plant Maintenance Engineering, 2011, 000(007):48-50.

4. B. Wang, Y.D. Wang, W.J. Liang, et al, Proceedings of the ASME, 2009, (06):26-30.

5. R.B. Zheng, X.G. Chen, Z.J. Chen et al, Journal of Gansu Sciences, 2017,29(6):117-120.

6. J.B.M. Juhani, R.B. Ibrahim, International Conference on Intelligent and Advanced 
Systems. IEEE, 2017:1-5.

7. L. Zhu, B. Zou, S.H. Gao et al, Advanced Materials Research, 2014, 1037 (24):169-173.

8. S.M. Jafari, H. Mehdigholi, M. Behzad, Shock and Vibration, 2014, (1):1-9.

9. H.D. Ma, R. He, D.J. Chen, Pressure Vessel Technology 2020, 37 (2):24-29.

10. X.G. Chen, X.H. Zhang, Journal of Gansu Sciences, 2018, 30(6):123-129.

11. H. Jing, L.B. Zhang, W. Liang, et al, Acta Petrolei Sinica, 2014, 35(1):172-177.

12. F. Van Herpe, ACTRAN user's conference 2010.Brussels, Belgium, 2010.

13. Y. Wang, S.X. Wang, Y.H. Liu, Journal of Ship Mechanics, 2012, 15(11):1321-1328. 